\title{
CONSUMO DE DIARIOS Y PARTICIPACIÓN ELECTORAL EN ESPAÑA (1977-2009)
}

\section{CONSUMPTION OF NEWPAPERS AND ELECTORAL PARTICIPATION IN \\ SPAIN (1977-2009)}

\author{
Javier Galán \\ Guadalupe Aguado \\ Josep M. Sanmartí \\ Raúl Magallón \\ Universidad Carlos III, Madrid. España/ Spain \\ javier.galan@uc3m.es \\ maguado@hum.uc3m.es \\ jsanmart@hum.uc3m.es \\ raul.magallon@uc3m.es
}

\begin{abstract}
"Si estuviera a mi alcance el decidir si deberíamos tener un gobierno sin periódicos o periódicos sin un gobierno, no dudaría un momento en preferir lo último. Pero debo decir que todo el mundo debería recibir esos periódicos, y ser capaz de leerlos" Thomas Jefferson
\end{abstract}

Recibido/Received: 05/05/2011

Aceptado/Accepted: 03/10/2011

\section{RESUMEN}

La influencia de los medios de comunicación en los procesos electorales y las decisiones de los ciudadanos ha motivado numerosos estudios desde mediados del siglo XX. A pesar de ello, subsisten estereotipos concretos como por ejemplo la correlación directa entre consumo de periódicos y participación electoral. Este artículo demuestra por comunidades autónomas que en España no existe esta supeditación directa y que se dan situaciones muy diversas. Destaca el caso de Castilla-La Mancha que es la comunidad autónoma con un mayor índice global de participación, y sin embargo con menor porcentaje en cuanto a difusión de diarios se refiere. El estudio pone de relieve otros aspectos importantes, como es el peso de los factores ideológicos a la hora de votar. En todo caso, cada vez es más complicado establecer una relación de influencia de los medios de comunicación en la decisión de los electores, debido al incremento general de las fuentes de información.

\section{PALABRAS CLAVE}

Influencia de los medios, elecciones, factores ideológicos, periódicos, Castilla-La Mancha.

\section{SUMARIO}

1. Introducción. 2. Relación medios de comunicación-procesos electorales. 3. Participación electoral en España. 4. Índices de difusión de diarios. 5. Participación política por comunidades autónomas. 6. Difusión de diarios por comunidades autónomas. 7. Relación entre comportamiento político y difusión de diarios. 8. Conclusiones y cuestiones a desarrollar. Bibliografia. 


\begin{abstract}
The influence of the media in electoral processes and decisions of citizens has motivated numerous studies since the mid-twentieth century. However, there are specific stereotypes such as the direct correlation between consumption of newspapers and electoral participation. This article shows through autonomous communities that in Spain there is not a direct subordination and very different situations are given. It highlights that Castilla-La Mancha is the region with a higher overall rate of participation, and yet with the lowest percentage of voters who buy a newspaper. The study underlines as well other issues, such as the weight of ideological factors when voting. In any case, there is increasingly difficulties to establish a relationship of influence of the media in the decision of the voters, because of the general increase in information sources.
\end{abstract}

\title{
KEYWORDS
}

Influence of media, elections, newspapers, ideological factors, Castilla-La Mancha.

\section{CONTENTS}

1. Introduction. 2. Relationship between media and elections. 3. Voter turnout in Spain. 4. Rates of diffusion. 5. Political participation through autonomous communities. 6. Dissemination of newspapers by autonomous communities. 7. Relationship between political behaviour and newspapers diffusion. 8. Conclusions and matters to develop. References.

\section{INTRODUCCIÓN}

El objetivo de este artículo es presentar los primeros resultados que ha obtenido este equipo investigador sobre las conexiones que se establecen entre consumo de medios, decisión de voto y campañas electorales. Estos primeros resultados se refieren a la relación que pueda existir entre difusión de diarios de información general y participación en las elecciones políticas celebradas en España.

En esta primera fase del estudio, se ha analizado si hay alguna vinculación directa y en qué grado, entre difusión de diarios y participación electoral, así como, en la medida de lo posible, establecer una relación de causalidad.

Para obtener los resultados se han tenido en consideración los índices de participación en todas las elecciones que se han celebrado en España desde el advenimiento de la democracia en 1977 hasta la elecciones europeas de 2009, hayan sido generales, autonómicas, municipales o europeas, sin tener en cuenta los referéndums celebrados, indistintamente de su ámbito, nacional, autonómico o local.

Se han estudiado dichos datos tanto a escala estatal como de comunidades autónomas, estableciendo comparativas de los procesos que se producen en cada una de ellas, de tal manera que se puedan determinar elementos comunes que permitan formular unas conclusiones, más o menos generales.

En segundo lugar, se han analizado las cifras de difusión de diarios que desde 1994 aporta la Información y Control de Publicaciones a través de su división OJD, también por comunidades autónomas, con el fin de relacionarlas con los datos de las elecciones y poder alcanzar las conclusiones pertinentes.

Establecida una base de datos con todas estas aportaciones, se aplicará un análisis por comunidades autónomas con el objetivo de buscar parámetros comunes. De esta manera, se 
han buscado nuevas vías para describir los factores que determinan la influencia de los diarios en la ciudadanía y en los procesos de decisión electoral.

La idea es aplicar, en posteriores fases de la investigación, la misma metodología a la radio, a la televisión y a Internet, para relacionar más adelante los datos con nuevas variables, y diseñar así un modelo de influencia de los medios de comunicación en el comportamiento político-electoral de la sociedad. Y, en el caso español, teniendo en cuenta el papel que ha jugado el Estado en el desarrollo del sistema de prensa (Aguado et al., 2009).

Finalizadas las fases, el objetivo siguiente es relacionar este modelo con el que se genera a través de los datos de las encuestas del CIS: los Barómetros de Opinión. Esto aportará los datos referidos a la percepción de los mensajes, de tal manera que se pueda establecer un modelo completo de estudio tanto en lo referente a los emisores como a los receptores.

\section{RELACIÓN MEDIOS DE COMUNICACIÓN- PROCESOS ELECTORALES}

Los efectos de los medios de comunicación en el comportamiento social han sido objeto de numerosos estudios y diversas teorías, que intentan refrendar el modo en que éstos influyen en el comportamiento político.

Dichas formulaciones justifican prácticamente todas y cada una de las opciones posibles: desde una influencia total en la que se sostiene que los medios mediatizan los comportamientos sociales, hasta el reconocimiento de que los medios no influyen para nada en las acciones sociales. Obviamente, también existe una serie de teorías que reconocen una cierta influencia pero de intensidad diversa, o que ésta depende de las circunstancias, sociales o personales, de cada uno de los receptores.

De Fleur lo explica de la siguiente manera: "No está claro en absoluto cual de las teorías explica mejor la relación entre los medios de comunicación y la gente que constituye las sociedades en las que se difunden los mensajes. Ninguna explicación sencilla predice de forma completa cómo va a ser esta relación. Además, algunas de las teorías se contradicen abiertamente unas con otras, ya que una dice que la influencia que reciben los miembros del público como resultado de su exposición a las comunicaciones de masas es inmediata, universal, directa e intensa, mientras que la otra mantiene que dichas influencia se producen a largo plazo, y son indirectas, selectivas y limitadas" (De Fleur, 2001:379). J. M. Sabucedo se refiere a ello argumentando: "Desde el momento de la aparición de los medios de comunicación de masas, una de las grandes cuestiones planteadas versa sobre los efectos que esos medios ejercen en la población. Las respuestas que se han dado a este problema en diferentes momentos pueden parecer, a primera vista, contradictorias: de la atribución de un poder casi absoluto en la determinación de la conducta política de las personas, a contemplarlos como meros elementos reforzadores de disposiciones y actitudes previas.

Pero este hecho no debe resultar sorprendente. Antes al contrario, es un fenómeno bastante extendido en la investigación social. La explicación del mismo suele ser bastante sencilla y responde, entre otras, a algunas de las siguientes causas: generalización no fundamentada en los resultados obtenidos en un estudio, olvido y/o marginación de variables que modulan los efectos observados, búsqueda de nuevas áreas en las que los fenómenos estudiados puedan ponerse de manifiesto, etc." (Sabucedo, 1977:12).

Un punto de inflexión en la evolución de las teorías que intentan explicar las relaciones entre sociedad y medios de comunicación fue la investigación The People's Choice realizada por 
Lazarsfeld, Berelson y Gaudet en 1944. En primer lugar, porque es un estudio empírico que estudia de manera cuantitativa cómo se establecían las influencias entre los distintos actores a la hora de determinar la decisión de voto, poniendo de manifiesto la necesidad de realizar estudios empíricos a la hora de estudiar los efectos de los medios en los receptores (Rospir, 2004:27-39). Y en segundo lugar, por las propias conclusiones a las que llegó. En ellas se puso de manifiesto que la influencia de los medios en el comportamiento político no era tanta como defendían las teorías en boga. No ha de olvidarse que las teorías predominantes de la época mantenían la influencia directa de los medios en el comportamiento político de la gente. De alguna manera sirvió para que se cambiase la orientación de muchas hipótesis y se buscase en otras causas las influencias de los medios.

De hecho, en ese estudio se manifiesta que "cada vez que a los encuestados se les pidió que informaran sobre sus contactos más recientes con comunicaciones electorales de todo tipo, las discusiones políticas fueron mencionadas con mayor frecuencia que el contacto con la radio o con el material impreso" (Lazarsfeld, Berelson y Gaudet, 1960:137).

Un estudio realizado por Weaver (a propósito de las elecciones presidenciales de Estados Unidos de 1976) indicaba que la prensa desempeña en los procesos electorales un papel central en la determinación de qué candidatos y qué rasgos específicos sobresalen y alcanzan una mayor visibilidad en relación al resto de elementos (Weaver; Graber; Mc Combs y Eyal, 1981). Según Weaver la canalización mediática influye de una manera muy importante, puesto que "al hacer que ciertos temas sobresalgan por encima del resto, los medios contribuyen de una manera significativa a la construcción de una realidad de la que luego dependerá la decisión de votar o no y por quién deben hacerlo en caso afirmativo. Asimismo, el que los medios dediquen una considerable cobertura al proceso electoral parece también contribuir a que el propio campo de la política destaque por encima de otras cuestiones de interés social, incrementando así el interés y potenciando la participación de la gente en las elecciones" (Weaver, 1997:234). Más recientemente Hallin y Mancini (2008) estudian las relaciones entre los sistemas de medios de comunicación y los sistemas políticos, para establecer tres modelos de relación entre los medios de comunicación y la política.

En los últimos años, los estudios que relacionan las campañas electorales y la participación ciudadana se han centrado en variables externas que configuran y delimitan el consumo de medios y no tanto en el consumo directo de los medios de comunicación, concretamente la prensa. Estas investigaciones se han orientado hacia el cambio de opinión que producen determinados acontecimientos narrados por los medios, el aumento del interés de los electores por adquirir información acerca de los hechos reseñables y su reflejo en el índice de participación (López García, 2007). Otros en cambio se ha referido al carácter ritual de la política y su capacidad para establecer un consenso identitario nacional (Hwa Ting, 2010), el papel de Internet en las campañas electorales (Macnamara, 2008); Xenos y Moy 2007), o la importancia de las redes sociales (Williams y Gulati, 2009).

El cambio de modelo comunicacional que ha generado el desarrollo de Internet y el asentamiento e implantación del uso de la redes sociales ha impulsado el debate y los estudios sobre la relación de la política y los medios, introduciendo nuevas perspectivas. Este debate sobre la participación se sustenta en otro más amplio sobre la crisis social y democrática, a la que muchos investigadores (Kaase y Newton, 1995; Klingeman y Fuchs, 1995; Norris, 1999) dan un alcance global. Y es que estos estudiosos hablan sobre todo de crisis de participación, como ocurre en los Estados Unidos, donde los ciudadanos aparecen cada vez menos implicados en los asuntos públicos (Putnam, 1995a, 1995b y 2000). No 
obstante, esta visión de Putnam de que estamos ante una crisis de participación ciudadana ha sido puesta en entredicho por otros analistas como Welzel, quien afirma que las generaciones más jóvenes no son menos participativas que las anteriores, sino todo lo contrario (Welzel et al., 2005). También Topf (1995) sostenía que con la excepción de España y Finlandia la acción política en sus distintos registros se había acrecentado en toda Europa desde los años 1980. Y de igual modo Gundelach (1995) concluía que la participación de base en sus diversos registros había crecido en la mayoría de países europeos, de nuevo con la salvedad de España. El caso español es puesto de relieve por Morales (2005: 53), cunado señala directamente que "el consenso general entre los analistas españoles es que la participación política y ciudadana ha disminuido en las últimas décadas en España". Al mismo tiempo se pregunta si este declive participativo en las sociedades postindustriales tiene el mismo significado en las democracias "jóvenes", como la española, que en aquellas que ya cuentan con una larga tradición histórica (2005: 54).

Este tipo de comportamientos pueden acarrear consecuencias serias para los sistemas democráticos, ya que en palabras de Bendit (1999) "la participación ciudadana (ya sea de jóvenes o de adultos) en procesos políticos, tanto a nivel local, regional, nacional o supranacional, debe ser considerada como un elemento central en el funcionamiento de los sistemas democráticos". Por lo mismo "el interés por la política y la participación activa, por otra parte, deben ser vistos tanto en su dimensión institucional, es decir en la disposición de los ciudadanos a participar en formas convencionales e institucionalizadas de actividad política (elecciones, afiliación a partidos políticos, candidaturas, mesas o colegios electorales, etc.), así como en la dimensión extrainstitucional, es decir en formas no convencionales y no institucionalizadas de participación, por ejemplo en forma de protestas, manifestaciones, peticiones, recolección de firmas, manifiestos, solicitudes en periódicos, etc.".

Más concretamente y referidos España se ha realizado diversos estudios sobre la relación de los medios con la política, especialmente en la época de la transición, como son los trabajos de Cavero (1991), Barrera (1995), Alférez (1996), Arroyo (2006), Vinuesa (2008) y Zugasti (2008), más enfocados en analizar cómo los medios de comunicación de aquella época, especialmente los diarios, ayudaron a construir el sentir democrático en la sociedad española y cómo se relacionaron con las fuerzas políticas.

Respecto a la influencia de los medios en las campañas electorales celebradas en España, Aceves (1993) ya realizó un estudio sobre las primeras de la España democrática; Bezunartea (2000) estudió el caso del País Vasco y más recientemente González (2003).

En esta línea presentamos estos primeros resultados en los que se estudia el modelo de España y, en concreto, la relación que existe en este sistema entre difusión de diarios y participación en las elecciones.

\section{PARTICIPACIÓN ELECTORAL EN ESPAÑA}

Para establecer los índices de participación en las elecciones se han revisado todas las celebradas en España desde el advenimiento de la democracia reciente:

- Elecciones Generales de los años 1977, 1979, 1982, 1986, 1989, 1993, 1996, 2000, 2004 y 2008. 
- Elecciones Autonómicas de los años 1983, 1987, 1991, 1995, 1999, 2003 Y 2007. En estos años se han incluido los datos de las elecciones correlativas a los Parlamentos de Andalucía, Cataluña, Galicia y País Vasco.

- Elecciones Locales de los años 1979, 1983, 1987, 1991, 1995, 1999, 2003 y 2007

- Elecciones al Parlamento Europeo de los años 1987, 1989, 1994, 1999, 2004 y 2009. Para obtener los datos se ha recurrido a diferentes fuentes. Para los referidos a las elecciones generales, municipales y al Parlamento Europeo se ha consultado la base "Historia de Resultados Electorales", del Ministerio del Interior (http://www.elecciones.mir.es). Para las elecciones municipales de 1979 y 1983 se han empleado dos ficheros facilitados desde el propio Ministerio del Interior. Los datos históricos de las elecciones autonómicas se encuentran normalmente en la web de los parlamentos correspondientes, pero no están organizados de una forma homogénea y con todos los parámetros necesarios para la realización de esta investigación. Para este tipo de elecciones se ha recurrido a los datos ofrecidos por la web del Congreso de los Diputados (http://www.congreso.es). Las cifras obtenidas en esta fuente han requerido una elaboración propia para obtener porcentajes. También hay que señalar que determinados datos de algunas elecciones no aparecen en esta web y han sido obtenidos en el "Archivo Histórico Electoral" del Gobierno Valenciano ( http://www.pre.gva.es/argos/archivo/).

En la relación de las comunidades autónomas estudiadas no se ha incluido a Ceuta y Melilla por dos razones. La primera de ellas, porque no adquirieron este rango hasta la mitad del período estudiado; y segunda, porque debido a su posición geográfica, se parecen más a una provincia que a una comunidad autónoma propiamente, lo que podría provocar una desviación considerable de los resultados en lo referente a la difusión de los diarios.

Establecidas estas fuentes y analizados los datos, se puede observar que históricamente, el mayor índice de participación se alcanzó en las elecciones generales del año 1982, con casi un 80 por ciento, mientras que el índice menor se halla en las elecciones europeas de 2004. Es destacable también señalar que en las dos ocasiones en las que las elecciones al Parlamento Europeo se han celebrado al mismo tiempo que las autonómicas y municipales (1987, 1999) los índices de participación han sido similares, siendo en estas ocasiones cuando más se ha votado en unas elecciones europeas.

\section{Gráfico 1. Histórico de la participación}

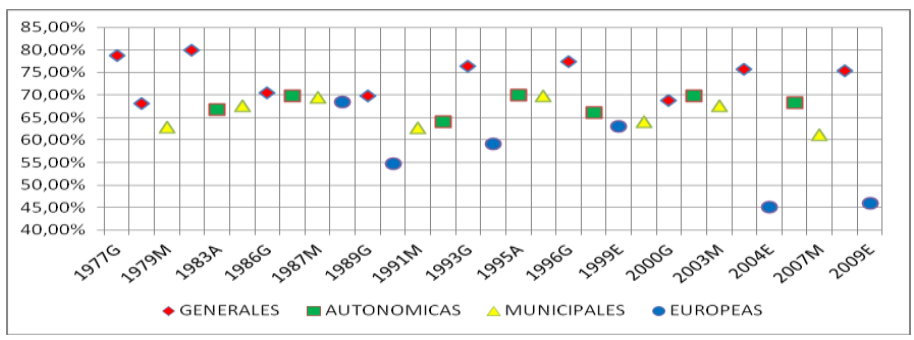

Fuente: Ministerio del Interior. Elaboración propia

En el Gráfico 1 se puede observar cómo los índices de participación en las elecciones varían considerablemente dependiendo del tipo y, a su vez, dentro de cada una de ellas la situación social concreta del momento también hace oscilar la participación. En este sentido, las 
elecciones generales siempre han tenido la participación más alta, excepto en los años 1989 y 2000 , en el que las autonómicas las superaron ligeramente. Por el contrario las elecciones europeas son las que muestran un menor índice de participación.

La participación media general en España en las elecciones celebradas desde 1977 hasta 2009 se sitúa en el 65,8 por ciento. Por tipo de elecciones, las generales se sitúan en un índice medio de participación del 74 por ciento, mientras que las autonómicas tienen un índice medio de participación del 67,82 por ciento. Las locales, un poco por debajo de esta cifra, muestran un índice de participación del 65,39 por ciento, en tanto que las europeas se quedan en un índice medio de participación del 58,77 por ciento, el más bajo de todas.

Tabla 1. Participación electoral

\begin{tabular}{|l|l|}
\hline Media de participación en las elecciones celebradas en España & $65,8 \%$ \\
\hline Elecciones generales que superan la media de participación & $100 \%$ \\
\hline Elecciones autonómicas que superan la media de participación & $85,7 \%$ \\
\hline Elecciones municipales que superan la media de participación & $50,0 \%$ \\
\hline Elecciones europeas que superan la media de participación & $0 \%$ \\
\hline
\end{tabular}

En cuanto a posibles tendencias, se puede observar que las elecciones generales muestran una pequeña tendencia al alza, mientras que en el caso de las autonómicas y municipales es hacia la baja. En lo que respecta a las elecciones europeas muestran una tendencia muy agudizada a la baja.

Este comportamiento de participación descrito a escala estatal se refleja en cada una de las comunidades autónomas, donde también las elecciones generales son las que cuentan con una mayor participación. Las autonómicas y municipales se encuentran por debajo, pero casi con el mismo índice. No obstante, en las autonomías en las que se celebran las elecciones autonómicas en fecha distinta a las locales, las elecciones municipales presentan un índice inferior a las autonómicas.

De estos datos se puede concluir que el componente ideológico juega un papel muy importante en la participación política electoral, ya que es indudable que las elecciones generales, en casi todas las comunidades autónomas, tienen una mayor carga ideológica que el resto de las elecciones, lo que se refleja en los mayores índices de participación. Por el contrario, el factor cercanía, el del gobierno más próximo al ciudadano, apenas influye en la participación. Por otra parte, estos datos también ponen de manifiesto que el gobierno europeo apenas ha calado en los votantes españoles.

\section{DIFUSIÓN DE DIARIOS}

Para los datos de difusión de diarios se ha utilizado la base de datos de Información y Control de Publicaciones, en concreto los correspondientes a su división OJD (https://www.introl.es/web_ojd/). En esta base de datos se han consultado los datos evolutivos del periodo por autonomías, considerando los siguientes parámetros:

Filtro geográfico: Autonomía.

Fecha: Desde enero de 1994 hasta diciembre de 2009. 
Modelo: Pago.

Periodicidad: Diaria.

Clasificación: Diarios de Información General.

Publicaciones: Diarios de Información General.

Canales: Difusión.

Se ha fijado el periodo temporal (1994-2009) para analizar la difusión de los diarios por ser el más homogéneo en cuanto a sistema de medición, aunque sea más corto que las series temporales de las elecciones. Téngase en consideración que "para realizar una auténtica labor al servicio de la publicidad, la OJD ha tenido que ir adaptando con los años su Reglamento de Trabajo, para dar cabida a las múltiples estrategias de comercialización de las cabeceras, hasta tal punto que en ese proceso de adaptación se ha visto forzada a establecer cambios en su propia estructura" (Aguado et al., 2008:253). De hecho en 1994 varió el procedimiento por el que se establece la difusión de los diarios. Por ello, se ha considerado que una serie temporal de catorce años es bastante adecuada para establecer deducciones suficientemente sólidas.

El trabajo se ha centrado en la difusión pagada, es decir, ejemplares vendidos, por ser el dato más significativo, ya que el acto de compra de prensa supone una mayor adhesión al producto informativo con su correspondiente influencia en el público. Han sido objeto de estudio los diarios de información general, no habiéndose tenido en cuenta los deportivos por su contenido específico, ni los de información económica por su escasa difusión. Tampoco se han analizado los gratuitos, ya que proporcionan una serie temporal muy corta. Como vemos en el Gráfico 2 esta cifra ha tenido diversas fluctuaciones a lo largo de los años.

A lo largo de estos años en España se han vendido diariamente una media de casi tres millones de diarios de información general. En este período de tiempo, la venta de diarios se ha incrementado en unos trescientos mil ejemplares, lo que representa una línea de tendencia al alza, si bien en los tres últimos años la tendencia es por el contrario claramente a la baja.

Gráfico 2. Evolución de la difusión de diarios en España

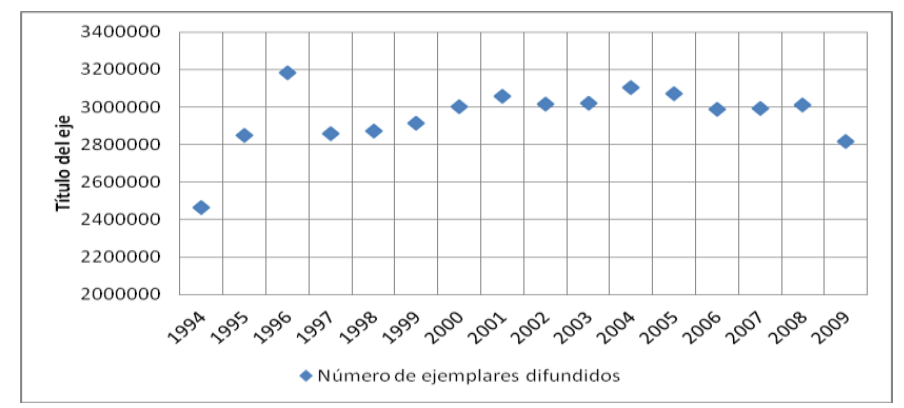

Fuente: OJD. Elaboración propia

Los años en los que más ejemplares de diarios se vendieron fueron en el 2000 y en el 2004. Y un dato interesante de resaltar es que en el año 2008 se vendieron casi los mismos ejemplares que en el año 1999, lo que pone de manifiesto que está industria, la de creación y venta de diarios en papel está claramente en crisis. 
Ahora bien, estos datos en sí mismos no son especialmente representativos, pues aunque muestran que se ha incrementado el número de ejemplares vendidos, también hay que tener en cuenta que la población en España ha ido aumentando en la última década.

Para obtener un dato más representativo hemos creado un índice de difusión en el que ponemos en relación el número de ejemplares difundidos con el de personas censadas en las elecciones generales del período.

Tabla 2. Ejemplares difundidos respecto a personas censadas

\begin{tabular}{|c|c|c|c|}
\hline $\begin{array}{l}\text { Ejemplares } \\
\text { difundidos }\end{array}$ & $\begin{array}{l}\text { Personas } \\
\text { censadas }\end{array}$ & $\begin{array}{l}\text { Porcentaje (por 1000) de diarios } \\
\text { difundidos respecto personas censadas }\end{array}$ \\
\hline 1996 & 3180705 & 32.531 .833 & 98 \\
\hline 2000 & 3003036 & 33.969 .640 & 88 \\
\hline 2004 & 3103074 & 34.571 .831 & 90 \\
\hline 2008 & 3012059 & 35.073 .179 & 86 \\
\hline
\end{tabular}

Fuente: Ministerio del Interior. OJD. Elaboración propia

De esta manera, la Tabla 2 ilustra sobre el tanto por mil de diarios difundidos respecto a las personas censadas, que bien podría interpretarse, como porcentaje de persona censadas que compran un diario de información general. Entre las conclusiones más relevantes, se puede señalar que en el año 1996, el 98 por mil del censo electoral era comprador de periódicos, mientras que en el año 2008 ese porcentaje descendió hasta el 86 por mil. Esta disminución pone una vez más de manifiesto la decreciente evolución en la difusión de los diarios y la crisis de esta industria.

Si se relacionan estos datos de difusión de ejemplares con los datos que hacen referencia al comportamiento electoral, se puede señalar que tanto la participación electoral como el porcentaje de diarios difundidos tienen una tendencia a la baja. Sin que se pueda establecer, de momento, una relación de causa-efecto entre ambas. No sería acertado afirmar que como se produce una bajada de número de censados que compren los diarios de información general, se origina una menor participación en la elecciones políticas. En cambio, lo que sí se puede afirmar sin riesgo a equivocarse es que en España cada vez se participa menos en las elecciones, y cada vez es menor el porcentaje de electores que compran diarios de información general.

\section{PARTICIPACIÓN POLÍTICA POR COMUNIDADES AUTÓNOMAS}

Como señalábamos con anterioridad, los índices de participación en el Estado se reflejan en el comportamiento político de cada una de las comunidades autónomas españolas. Siendo eso así, cada una de ellas tiene algunas peculiaridades que vamos a explicar con más detalle. En primer lugar, en todas las comunidades autónomas, el mayor índice de participación corresponde a las elecciones generales, y el menor índice a las europeas.

$\mathrm{Si}$ tomamos como referencia la media general de participación de todas las elecciones celebradas se puede decir que Castilla-La Mancha es la comunidad autónoma que más vota, liderando un grupo formado por Extremadura, Cantabria, Comunidad Valenciana, La Rioja, 
Gráfico 3. Media de participación, por tipo de elecciones, por Comunidades Autónomas

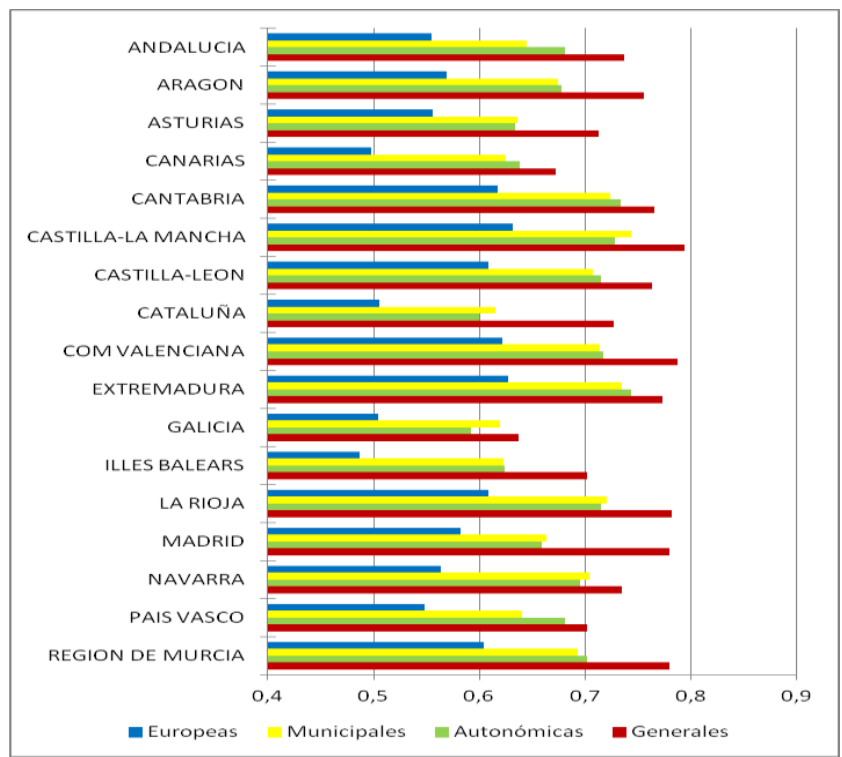

Fuente: Ministerio del Interior. Elaboración propia.

Castilla-León y Región de Murcia, que presentan un índice de participación por encima de la media, y que además también están por encima de la media de participación en cada tipo de elecciones.

Gráfico 4. Media de participación por Comunidades Autónomas

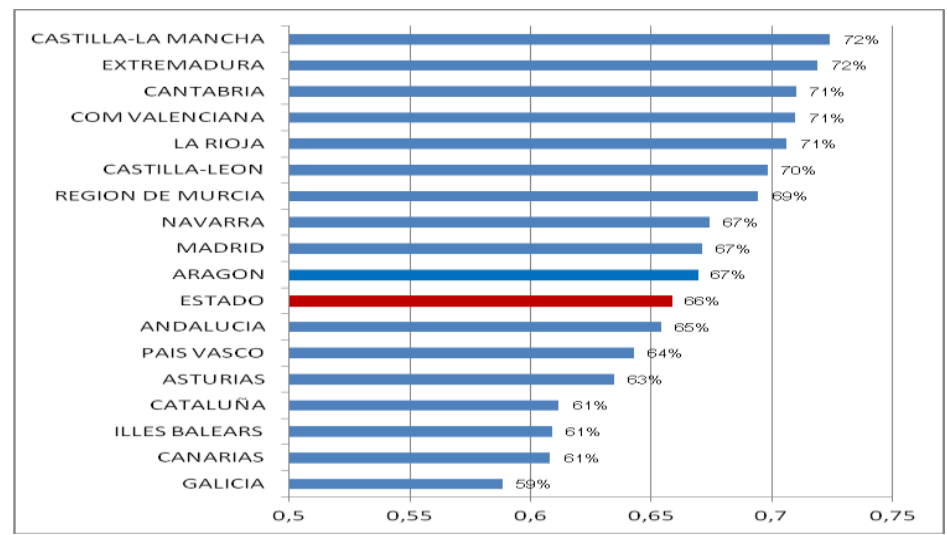

Fuente elaboración propia

Les sigue un grupo formado por Navarra, Aragón y Madrid, que estando por encima de la media en cuanto a índice global de participación, no se sitúan por encima de la media en cada uno de los distintos tipos de elecciones. Navarra no está por encima de la media ni en las 
elecciones generales, ni en las europeas, pero sí en las autonómicas y en las municipales. Aragón está por encima de la media en las elecciones generales y municipales, pero no en las autonómicas y europeas. Madrid participa por encima de la media en las elecciones generales y en las europeas, pero no en las autonómicas, ni en las municipales.

Luego se encuentran Andalucía y País Vasco, que están por debajo de la media de participación en todas las elecciones, excepto en las autonómicas.

El resto de las comunidades autónomas está por debajo de la media global y por debajo de la media de participación en cada uno de los grupos de elecciones. Este grupo está formado por Asturias, Cataluña, Canarias, Illes Balears y Galicia, que es la comunidad autónoma con menor índice de participación en todo tipo de elecciones.

Tabla 4. Participación en las comunidades autónomas

\begin{tabular}{|l|l|}
\hline Autonomías que superan la participación media del Estado & $58,8 \%$ \\
\hline Autonomías con una participación por debajo de la media del Estado & $41,2 \%$ \\
\hline Autonomías en las que la participación más alta es en las elecciones generales & $100 \%$ \\
\hline $\begin{array}{l}\text { Autonomías en las que las participación en las elecciones autonómicas es mayor } \\
\text { que en las municipales }\end{array}$ & $58,8 \%$ \\
\hline $\begin{array}{l}\text { Autonomías en las que las participación en las elecciones municipales es mayor } \\
\text { que en las autonómicas }\end{array}$ & $41,2 \%$ \\
\hline Autonomías en las que la participación más baja es en las elecciones europeas & $100 \%$ \\
\hline
\end{tabular}

\section{DIFUSIÓN DE DIARIOS POR COMUNIDADES AUTÓNOMAS}

Siguiendo con la hipótesis planteada, en este apartado se analizan los datos de difusión de los diarios por comunidades autónomas para posteriormente ponerlos en relación con los índices de participación política con el objetivo de buscar conclusiones significativas.

Tabla 5. Promedio de ejemplares vendidos por Comunidad Autónoma

\begin{tabular}{|c|c|}
\hline Andalucía & 353109 \\
\hline Aragón & 81078 \\
\hline Asturias & 112870 \\
\hline Canarias & 125797 \\
\hline Cantabria & 56878 \\
\hline Castilla-León & 176231 \\
\hline Castilla-La Mancha & 48729 \\
\hline Cataluña & 556336 \\
\hline Comunidad Valenciana & 265120 \\
\hline Extremadura & 46095 \\
\hline Galicia & 223795 \\
\hline Illes Balears & 62926 \\
\hline La Rioja & 24992 \\
\hline Madrid & 441274 \\
\hline Navarra & 81722 \\
\hline País Vasco & 239269 \\
\hline Region de Murcia & 55112 \\
\hline
\end{tabular}

Fuente: OJD. Elaboración propia. 
Por comunidades autónomas el número de ejemplares de diarios de información general vendidos anualmente es muy dispar, entre otras razones por la diferente población que tiene cada una de ellas.

Desde esta perspectiva, se puede señalar que es en Cataluña donde mayor número de diarios se vende, por encima de los quinientos mil ejemplares de media diaria. En segunda posición se encuentra Madrid, con una media que supera los cuatrocientos mil ejemplares, seguida por Andalucía, con una media por encima de los trescientos mil ejemplares.

Por encima de los doscientos mil están Galicia y el País Vasco. Y superando los cien mil ejemplares se sitúan Asturias, Canarias, Castilla-León y Comunidad Valenciana. Por debajo de los cien mil ejemplares se halla el resto de comunidades autónomas.

Ahora bien, si se relacionan estas cifras con el número de personas censadas, se obtiene un índice de difusión más representativo. Es preciso señalar la importancia de este dato por ser considerado clave para establecer una posible relación entre difusión de diarios y comportamiento político.

Gráfico 5. Ejemplares difundidos por mil electores censados

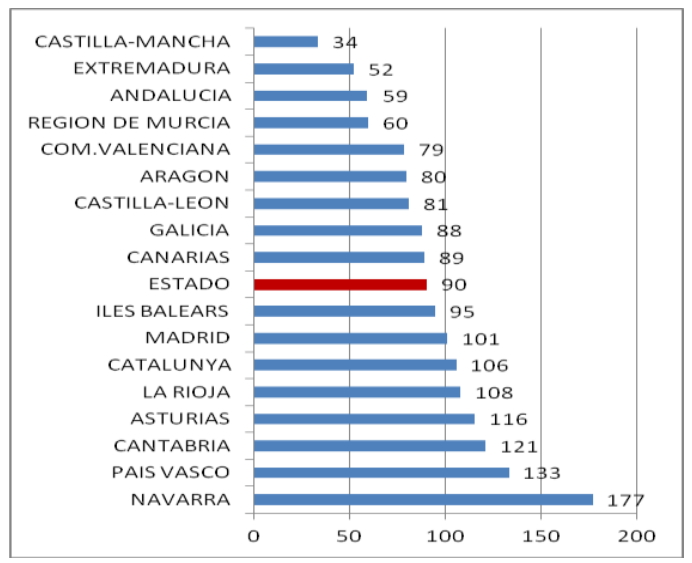

Fuente: Ministerio del Interior. OJD. Elaboración propia.

De esta forma, podemos observar cómo Navarra es la comunidad autónoma donde hay un mayor porcentaje de diarios difundidos por electores censados, liderando un grupo de ocho comunidades que tienen un porcentaje superior a la media. Por otra parte, Castilla-La Mancha es la comunidad autónoma con menor porcentaje de electores que compran un diario.

Tabla 6: Índice de difusión de diarios

\begin{tabular}{|l|l|}
\hline $\begin{array}{l}\text { Autonomías con un índice de difusión de diarios } \\
\text { superior a la media del Estado }\end{array}$ & $47,0 \%$ \\
\hline $\begin{array}{l}\text { Autonomías con un índice de difusión de diarios } \\
\text { inferior a la media del Estado }\end{array}$ & $53,0 \%$ \\
\hline
\end{tabular}




\section{RELACIÓN ENTRE COMPORTAMIENTO POLÍTICO Y DIFUSIÓN DE DIARIOS}

Por una parte, se han elaborado tablas que muestran el índice de participación media política por comunidades autónomas, que nos permiten saber en qué áreas geográficas se vota más y en cuáles menos. Describimos de esta manera el comportamiento político, en cuanto a participación en cada una de ellas. Por otra parte, se han elaborado tablas que reflejan qué comunidad autónoma tiene mayor o menor índice de difusión de diarios. Si se relacionan estos datos, se podrá concluir si en aquellas comunidades que tienen un mayor porcentaje de compradores de diarios, se vota más o se vota menos, y viceversa.

Tabla 7: Media de participación e índice de difusión

\begin{tabular}{|l|l|l|}
\hline & Participación & Difusión /000 \\
\hline Castilla-La Mancha & $\mathbf{7 2 , 4 1 \%}$ & 34 \\
\hline Extremadura & $\mathbf{7 1 , 9 1 \%}$ & 52 \\
\hline Cantabria & $\mathbf{7 0 , 9 9 \%}$ & $\mathbf{1 2 1}$ \\
\hline Comunidad Valenciana & $\mathbf{7 0 , 9 7 \%}$ & $\mathbf{7 9}$ \\
\hline La Rioja & $\mathbf{7 0 , 6 2 \%}$ & $\mathbf{1 0 8}$ \\
\hline Castilla-León & $\mathbf{6 9 , 8 2 \%}$ & 81 \\
\hline Region de Murcia & $\mathbf{6 9 , 4 3 \%}$ & 60 \\
\hline Navarra & $\mathbf{6 7 , 4 1 \%}$ & $\mathbf{1 7 7}$ \\
\hline Madrid & $\mathbf{6 7 , 0 9 \%}$ & $\mathbf{1 0 1}$ \\
\hline Aragón & $\mathbf{6 6 , 8 9 \%}$ & 80 \\
\hline Estado & $65,81 \%$ & 90 \\
\hline Andalucía & $65,40 \%$ & 59 \\
\hline País Vasco & $64,26 \%$ & $\mathbf{1 3 3}$ \\
\hline Asturias & $63,46 \%$ & $\mathbf{1 1 6}$ \\
\hline Cataluña & $61,17 \%$ & $\mathbf{1 0 6}$ \\
\hline Illes Balears & $60,89 \%$ & $\mathbf{9 5}$ \\
\hline Canarias & $60,82 \%$ & 89 \\
\hline Galicia & $58,82 \%$ & 88 \\
\hline
\end{tabular}

Fuente: Ministerio del Interior. OJD. Elaboración propia. En negrita las comunidades autónomas que están por encima de la media.

En los resultados de la tabla anterior puede apreciar que de las diez comunidades autónomas que tienen un índice de participación por encima de la media, seis de ellas tienen un índice de difusión de diarios por debajo de la media, mientras que las otras cuatro están por encima de la media. Y que las dos comunidades autónomas que tienen el índice de participación más alto, son las que tienen un índice de difusión de difusión de diarios más bajo. Lo que implica que un alto índice de participación electoral, no lleva aparejado necesariamente un alto índice de difusión de diarios. Pero tampoco puede afirmarse con rotundidad lo contrario, que un alto índice de participación lleve parejo un bajo índice de difusión de diarios, ya que no se cumple en todos los casos.

En segundo lugar puede observarse que de las siete comunidades autónomas que tienen un índice de participación política por debajo de la media; es decir, votan menos, cuatro de ellas tienen un índice de difusión de diarios superior a la media, y tres tienen un índice inferior a la 
media. Estos datos apuntarían a que se pudiera afirmar que en las comunidades autónomas donde se leen más diarios, se participa menos en las elecciones, pero tampoco se puede afirmar con rotundidad ya que, aunque se cumpla en la mayoría de la comunidades autónomas de mayor participación política, no se cumple en todas.

Tabla 8: Relación entre participación e índice de difusión

\begin{tabular}{|l|l|}
\hline $\begin{array}{l}\text { Autonomías que tienen una alta participación y un alto } \\
\text { índice de difusión }\end{array}$ & $24,5 \%$ \\
\hline $\begin{array}{l}\text { Autonomías que tienen una alta participación pero un } \\
\text { bajo índice de difusión }\end{array}$ & $35,2 \%$ \\
\hline $\begin{array}{l}\text { Autonomías que tienen una baja participación pero un } \\
\text { alto índice de difusión }\end{array}$ & $24,5 \%$ \\
\hline $\begin{array}{l}\text { Autonomías que tienen una baja participación y un } \\
\text { bajo índice de difusión }\end{array}$ & $17,6 \%$ \\
\hline
\end{tabular}

Estos datos permiten apuntar que no existe una relación clara entre la difusión de diarios de información general y participación política de los ciudadanos. Si se puede establecer que mayor difusión de diarios no implica necesariamente una mayor participación en las elecciones políticas, más bien, aunque de manera no concluyente, estos datos apuntan lo contrario ya que el mayor porcentaje corresponde al grupo de autonomías que tienen un alto índice de participación pero un bajo índice de difusión de diarios.

\section{CONCLUSIONES Y CUESTIONES A DESARROLLAR}

Primero. Del análisis realizado, se puede señalar que el componente ideológico sigue jugando un papel muy importante en la participación política. De este modo, y considerando que las elecciones generales tienen una mayor carga ideológica que el resto de comicios, podemos comprender que sean los que reflejen mayores índices de participación. En dicho sentido, se puede apreciar cómo en todas las comunidades autónomas el mayor índice de participación corresponde a las elecciones generales, y el menor corresponde a las europeas.

Segundo. La baja participación en los comicios europeos se puede explicar en cuanto se establece no tanto en relación al factor ideológico sino a los intereses nacionales. En este sentido, hay un déficit comunicativo por parte de partidos políticos y medios de comunicación a la hora de involucrar a la ciudadanía en las decisiones que se toman en el Parlamento Europeo. Mientras que las elecciones generales forman parte de una campaña permanente, el interés de los medios de comunicación y de los partidos políticos por las elecciones de ámbito europeo sólo aparece (y de manera secundaria) cuando se acerca la fecha de los comicios. Y, por otro lado, se refieren básicamente a asuntos y líderes nacionales, no europeos, con lo que se convierten en una especie de duplicado de las elecciones legislativas.

Tercero. Castilla-La Mancha es la comunidad autónoma con un mayor índice global de participación, y sin embargo es la comunidad autónoma con menor índice de difusión de diarios, mientras que Navarra es la comunidad autónoma donde mayor número de 
compradores diarios (178 por mil), y está entre las comunidades autónomas con mayor participación en comicios autonómicos y municipales. Es probable que se deba a la incidencia de los diarios locales. En ese sentido, habría que profundizar en la relación que puede existir entre diarios locales e intención de voto.

Cuarto. Navarra, Madrid y La Rioja son las comunidades autónomas en las que el índice de participación y difusión de diarios tiene una correlación más clara. De este modo, se puede señalar que existe una cierta vinculación entre comunidades autónomas de una sola provincial, índice de participación e índice de difusión de diarios.

Quinto. Además de las conclusiones que se pueden sacar en este análisis, en posteriores estudios habría que profundizar sobre la conexión entre la difusión de diarios y el voto emitido, así como en el estudio de la influencia que puedan tener los diarios regionales frente a los de difusión nacional, además de la incidencia que puede tener la pertenencia o no de un diario a un grupo empresarial determinado. Obviamente, dichos datos habría que complementarlos con las cifras referidas a audiencias de radio y televisión, y también con el consumo y utilización de los medios digitales.

Sexto. Cada vez es más complicado establecer una relación de influencia de los medios de comunicación en la decisión de los electores, debido al incremento general de las fuentes de información. En este sentido es necesario destacar que, al contrario de lo que pudiera pensarse, la auto-referencialidad de los medios hace que el consumo de información aumente de forma irregular hasta convertirlos en mensajes contradictorios.

Séptimo. Un índice representativo a desarrollar en el futuro será el que sepa relacionar el número de "ejemplares vendidos" respecto al número de personas con derecho a voto (censo electoral). Para ello habrá que tener en consideración las relaciones entre prensa nacional, prensa local y prensa regional.

\section{BIBLIOGRAFÍA}

ACEVES, F. (1993): "La influencia de los medios de comunicación en los procesos electorales". En Comunicación y Sociedad, № 18-19, pp. 223-255.

AGUADO, G., GALÁN, J., BEAUMONT, J. y GARCÍA, J.L. (2008): Organización y gestión de la empresa informativa, Madrid, Síntesis.

AGUADO, G., SANMARTÍ, J.M. y MAGALLÓN ROSA, R. (2009): "España, Francia, Italia y Portugal: cuatro modelos diferenciados de prensa euromediterránea", Revista Observatorio (OBS*), Vol. 3, No 2, disponible en: http://www.obs.obercom.pt/index.php/obs/article/view/233

ALFÉREZ, A. (1996): El cuarto poder en España. La prensa desde la Ley de Fraga a 1996, Barcelona, Plaza\&Janes.

ARROYO, M. y ROEL, M. (2006): Los medios de comunicación en la democracia (1982-2005), Madrid, Fragua.

BARRERA, C. (1995): Sin mordaza. Veinte años de prensa en democracia, Temas de Hoy, Madrid. BENDIT, R. (1999): "Participación social y política de los jóvenes en los países de la Unión Europea". Ponencia presentada durante la reunión del Grupo de Trabajo "Juventud”, Buenos Aires, Clacso. 
BEOBIDE EZPELETA, I. Ma (1999): "Prensa, Partidos Políticos y Elecciones Generales del 15 de junio de 1977", Estudios de Deusto: revista de la Universidad de Deusto, Vol. 47, No. 2, pp. 11-82.

BEZUNARTEA. O. y DEL HOYO, M. (2000): "Urnas y quioscos: dos espejos discordantes: dónde se refleja la sociedad vasca, en las preferencias que marca el consumo de prensa o en las que arrojan los resultados electorales". En Estudios sobre el Mensaje Periodístico. №. 6, pp. 109-134.

DE FLEUR, M. L. (2001): Teorías de la comunicación de masas, Barcelona, Paidós.

FERNÁNDEZ, G. (2000): "Notas sobre la participación política de los jóvenes chilenos", en La participación social y política de los jóvenes en el horizonte del nuevo siglo, BLARDINI, S. (coord.), Buenos Aires, Clacso, pp. 87-108.

GONZÁLEZ, M. (2003): "El papel de los medios de comunicación en la campaña electoral". En Ámbitos. No. 9-10, pp. 471-484.

GUNDELACH, P. (1995): "Grass-Roots Activity", en VAN DETH, J.W. y SCARBROUGH, E. (eds.): The Impact of Values. Nueva York, Oxford University Press.

HALLIN, D. y MANCINI, P. (2008): Sistemas mediáticos comparados, Madrid, Hacer Editorial.

HWA TING, M. (2010): "Ritual and Identity. Elections and Voting in Singapore". Taiwan Journal of Democracy, Volume 6, No. 2.

IBARRA, P. e IDOYAGA, P. (1998): "Racionalidad democrática, transmisión ideológica y medios de comunicación”, ZER n ${ }^{\circ}$ 5, pp. 157-181.

KAASE, M. y NEWTON, K.. (1995): Beliefs in Government, Nueva York, Oxford University Press.

KLAPPER, J. T. (1974): Efectos de las comunicaciones de masas, Madrid, Aguilar.

KLINGEMANN, H.D. y FUCHS, D. (eds.) (1995): Citizens and the State, Oxford, Oxford University Press.

LÓPEZ GARCÍA, G. (2007): "Polls, Public Demonstrations, Election Results, and the Media: The Events of March 11 in Spain". Zer. N ${ }^{\circ} 20$

MACNAMARA, J. (2008): "Internet media and the public sphere: The 2007 Australian electioneering experience". Media International Australia, no. 129, November, 7-19.

MENDOZA ELVIRA, G. (2006): "El papel de los medios de comunicación masiva en las elecciones", Revista de la Facultad de Derecho de México, №. 245, pp. 119-131.

MORALES, L. (2005): “¿Existe una crisis participativa? La evolución de la participación política y el asociacionismo en España”: Revista Española de Ciencia Política, 13, octubre 2005, pp. 51-87.

NORRIS, P. (1999): "Conclusions: The Growth of Critical Citizens and Its Consequences", en Norris, P. (ed.) Critical Citizens. Oxford, Oxford University Press.

PUTNAM, R. (1995a): "Bowling Alone: America's Declining Social Capital”, Journal of Democracy, 6: 65-78.

- (1995b): "Tuning In, Tuning Out: The Strange Disappearance of Social Capital in America", Political Science and Politics, 28: 664-683.

- (2000): Bowling Alone. The Collapse and Revival of American Community. Nueva York, Simon and Schuster.

SABUCEDO, J.M. y RODRÍGUEZ, M. (1977): Medios de comunicación de masas y conducta política, Madrid, Biblioteca Nueva.

TOPF, R. (1995): "Beyond Electoral Participation": Hans-Dieter Klingemann y Dieter Fuchs, eds., Citizens and the State. Nueva York, Oxford University Press.

VINUESA, M.L. (1997): Opinión pública y cultura política en la España democrática. Un estudio empírico de las elecciones generales de 1993. Madrid, Servicio Publicaciones UCM.

- (2008): "La opinión pública medida y mediatizada. La comunicación social en la España democrática", en Estudios sobre el Mensaje Periodístico, № 14.

WEAVER, D.H. (1997): "Canalización mediática ("agenda-setting”) y elecciones en Estados Unidos": CIC, $\mathrm{n}^{\circ} 3$, pp. 229-241, Madrid, Servicio de Publicaciones, UCM.

WEAVER, D. H.; GRABER, D.A.; McCOMBS, M. y EYAL, CH (1981): Media Agenda-Setting in a Presidential Election: Issues, Images and Interest, New York, Praeger. 
WELZEL, Ch.; RONALD I.; DEUTSCH, F. (2005): "Social Capital, Voluntary Associations, and Collective Action: Which Aspects of Social Capital Have the Greatest "Civic" Payoff?", Journal of Civil Society, 1.

WILLIAMS, C. Y GULATI, G. J. (2009): "Facebook Grows Up: An Empirical Assessment of its Role in the 2008 Congressional Elections", 2009 Annual Meeting of the Midwest Political Science Association, Chicago, IL.

WOLF, M. (1994): Los efectos sociales de los media, Barcelona, Paidós.

XENOS, M, y MOY, P. (2007): "Direct and Differential Effects of the Internet on Political and Civic Engagement", Journal of Communication, vol. 57, no. 4, 704-18.

ZUGASTI, R. (2008): "El papel de la prensa en la construcción de las democracia española", en Confines, de Relaciones Internacionales y Ciencia Política, $\mathrm{N}^{\mathrm{o}}$, 4-7, p: 53

\section{Breve currículo:}

\section{Javier Galán Gamero}

Profesor Titular de Periodismo de la Universidad Carlos III de Madrid. Doctor en Ciencias de la Información por la Universidad de Laguna. Co-director del Máster Periodismo Científico y Comunicación de la Ciencia. Coordinador de los Proyectos Fin de Grado de la Facultad de Humanidades, Comunicación y Documentación de la Universidad Carlos III de Madrid. Sus áreas de investigación se centran en la industria de la información, la comunicación en las organizaciones y la comunicación social de la ciencia, temas en los que ha publicado diversos artículos de investigación.

\section{Guadalupe Aguado}

Profesora del Departamento de Periodismo y Comunicación Audiovisual de la Universidad Carlos III de Madrid. Profesora Invitada en la Universidade de Coimbra (2007), Université de Paris II-La Sorbonne (2008), y University of California-San Diego (2010). Es autora de seis monografias dedicadas al análisis de medios de comunicación y coautora del manual Organización y Gestión de la Empresa Informativa (Síntesis). Ha publicado numerosos artículos en revistas de referencia investigadora en el ámbito de la comunicación.

\section{Josep M. Sanmartí Roset}

Doctor en Relaciones Internacionales por la Universidad Complutense de Madrid y profesor del Departamento de Periodismo y Comunicación Audiovisual de la Universidad Carlos III de Madrid. Profesor visitante en la Université de Paris II-La Sorbonne (2008), la University of California-San Diego (2010) y la University of Sydney (2011).

\section{Raúl Magallón Rosa}

Doctor Europeo por la Universidad Complutense de Madrid y profesor en el Departamento de Periodismo y Comunicación Audiovisual de la Universidad Carlos III de Madrid. Profesor Visitante en la Universidad París II-La Sorbonne (2008), Universidad de Leeds (RU) (2009), y la Universidad de California-San Diego (2010). 